\title{
SHELL-MODEL CALCULATIONS ON THE NICKEL ISOTOPES
}

\author{
P. W. M. GLAUDEMANS, M. J. A. DE VOIGT ${ }^{\dagger}$ and E F M. STEFFENS \\ Fysisch Laboratorium, Rujksuniversiteit, Utrecht, the Netherlands
}

Received 1 August 1972

(Revised 4 September 1972)

\begin{abstract}
Binding and excitation energies, electromagnetic transition rates and multipole moments have been calculated for ${ }^{57-66} \mathrm{~N} 1$ from many-particle shell-model wave functions with neutrons in the $2 p_{\frac{3}{2}}, 1 f_{\frac{5}{2}}$ and $2 p_{\frac{1}{2}}$ orbits outside the ${ }^{56} \mathrm{~N}_{1}$ core The effective two-body matrix elements are obtained from the modified surface delta interaction The average absolute deviation between the calculated and experimental binding and excitation energies is $0.11 \mathrm{MeV}$ (with the exclusion of ${ }^{66} \mathrm{~N}_{1}$ ) Electric quadrupole transition rates and moments are calculated with an effective neutron charge $e_{\mathrm{n}}=(170 \pm 008) e$, obtained from a least-squares fit to experimental data Magnetic dipole transition rates and moments follow from five fitted effective reduced singleparticle matrix elements. The average absolute deviations between theory and experiment for the strongest $\mathrm{E} 2$ transitions (1-15 W u $)$ and for the strongest M1 transitions (0.01-0 14 W.u.) are 35 and $003 \mathrm{Wu}$, while the average measured strengths are 70 and $0.07 \mathrm{Wu}$, respectively
\end{abstract}

\section{Introduction}

The structure of the Ni 1sotopes has already been studied with different models and interactions. Detailed shell-model calculations with neutrons in the $2 p_{\frac{3}{2}}, 1 f_{\frac{5}{2}}$ and $2 p_{\frac{1}{2}}$ orbits have been performed ${ }^{1,2}$ ) with effective-interaction matrix elements, obtained from least-squares fits to observed excitation energies. Spectra obtained with the freenucleon potential of Hamada and Johnstone are given in ref. ${ }^{3}$ ). The effective surface delta interaction (SDI) has been applied by Arvieu et al. ${ }^{4}$ ) and Plastino et al. ${ }^{5}$ ). The results of shell-model and quasiparticle calculations with several interactions are presented in refs. ${ }^{6-10}$ ). The use of Hartree-Fock-Bogoliubov wave functions is discussed in refs. ${ }^{11,12}$ ).

In these studies a systematic treatment of the electromagnetic properties of the $\mathrm{N}_{1}$ isotopes including also M1 decay is missing Since also new experimental data became avalable recently, it was thought worthwhile to study the level schemes and electromagnetic properties of the $A=57-66 \mathrm{~N} 1$ 1sotopes by means of shell-model calculations. In the present work the modified ${ }^{13}$ ) surface delta interaction (MSDI) has been used with ${ }^{56} \mathrm{~N}_{1}$ acting as an inert core The neutrons are allowed to occupy all possible configurations in the $2 \mathrm{p}_{\frac{3}{2}}, 1 \mathrm{f}_{\frac{5}{2}}$ and $2 \mathrm{p}_{\frac{1}{2}}$ orbits. It 1 assumed that possible core excitations ${ }^{14}$ ) can be taken into account implicitly by the effective interaction and (for transition rates) by the effective reduced single-particle transition matrix elements. It has been pointed out previously ${ }^{1,7,8}$ ) that the inclusion of the $\lg _{\frac{2}{2}}$ orbit in the calculations does not provide much better results.

† Present address University of Rochester, NSRL, Rochester, NY 14627. 
In the present work M1 and E2 transition rates are calculated for the lowest 5-10 states that are experimentally known in the $A=58-65 \mathrm{~N}_{1}$ isotopes and for three states in ${ }^{57} \mathrm{~N}_{1}$ and ${ }^{66} \mathrm{~N}$.

\section{Binding energies and level schemes}

The antisymmetrized two-body matrix elements of the modified surface delta interaction are given by ${ }^{13,15}$ )

$$
\begin{aligned}
& \left\langle j_{a} j_{b}|V| j_{c} J_{d}\right\rangle_{J T}=\frac{A_{T}}{2(2 J+1)}\left[\frac{\left(2 j_{a}+1\right)\left(2 j_{b}+1\right)\left(2 j_{c}+1\right)\left(2 j_{d}+1\right)}{\left(1+\delta_{a b}\right)\left(1+\delta_{c d}\right)}\right]^{\frac{1}{2}} \\
& \times\left\{(-1)^{l_{a}+l_{c}+J_{b}+j_{d}}\left\langle j_{b}-\frac{1}{2} j_{a} \frac{1}{2} \mid J 0\right\rangle\left\langle j_{d}-\frac{1}{2} j_{c} \frac{1}{2} \mid J 0\right\rangle\left[1-(-1)^{l_{c}+l_{d}+J+T}\right]\right. \\
& \left.-\left\langle j_{b} \frac{1}{2} j_{a} \frac{1}{2} \mid J 1\right\rangle\left\langle J_{d} \frac{1}{2} j_{c} \frac{1}{2} \mid J 1\right\rangle\left[1+(-1)^{T}\right]\right\}+B[2 T(T+1)-3],
\end{aligned}
$$

where $J$ and $T$ are the spin and isospin of the two-particle system, $j_{k}$ and $l_{k}$ the spin and the orbital angular momentum of a particle in orbit $k$, and $\left\langle j_{1} m_{1} j_{2} m_{2} \mid J M\right\rangle$ is a Clebsch-Gordan coefficient. For the given numerical expression of the MSDI matrix elements the coupling order $j=l+s$ (rather than $j=s+l$ ) 1 s assumed and the

TABLE 1

Binding energies (in MeV) for the ground states of the $\mathrm{N}_{1}$ isotopes with respect to ${ }^{56} \mathrm{~N}_{1}{ }^{\text {a }}$ )

\begin{tabular}{cccc}
\hline Mass & $\left.E_{b}(\exp )^{b}\right)$ & $E_{b}$ (calc) & Difference \\
\hline 57 & 1027 & 1021 & 006 \\
58 & 22.47 & 22.37 & 010 \\
59 & 31.47 & 3152 & -005 \\
60 & 4286 & 4279 & 007 \\
61 & 5068 & 50.74 & -006 \\
62 & 6127 & 61.24 & 003 \\
63 & 6812 & 68.27 & -0.15 \\
64 & 77.77 & 77.76 & 001 \\
65 & 8387 & 8380 & 007 \\
66 & $\left.9287^{c}\right)$ & 92.35 & 0.52
\end{tabular}

a) The binding energy of ${ }^{56} \mathrm{~N}_{1}$ is taken to be $48401 \mathrm{MeV}$ [ref ${ }^{16}$ )].

b) Ref. ${ }^{16}$ ).

c) This value has not been used in the fit

radial wave functions are taken positive near the origin. The interaction contans two adjustable parameters 1.e. the 1sospin dependent strength $A_{T}$ and the strength of the monopole term $B$. Since the active nucleons concerned are all neutrons, only $T=1$ matrix elements are required. The best values for the MSDI strengths and the singleparticle binding energies given by $A_{1}=0.537, B=0.469, E\left(\mathrm{p}_{\frac{3}{2}}\right)=-10.21$, $E\left(\mathrm{f}_{\frac{s}{2}}\right)=-9.55$ and $E\left(\mathrm{p}_{\frac{1}{2}}\right)=-9.25 \mathrm{MeV}$, follow from a least-squares fit to 36 observed excitation energies and the 9 ground state binding energies of the $A=57-65$ Ni isotopes. 
For the ground state binding energies of these 1sotopes given in table 1, a very good agreement with experımental data ${ }^{16}$ ) is obtained with an average deviation of only $0.07 \mathrm{MeV}$. A rather strong deviation occurs for ${ }^{66} \mathrm{~N}$. The differences between ground

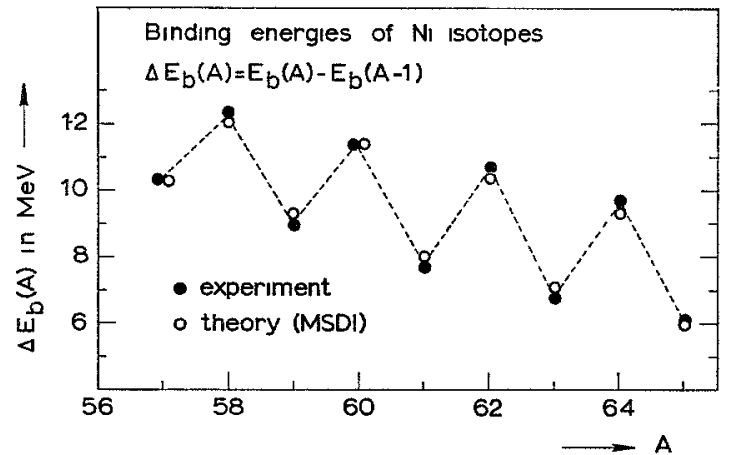

Fig 1. A comparison between theory and experiment for the binding energy differences for the ground states of $\mathrm{N}_{1}$ isotopes.

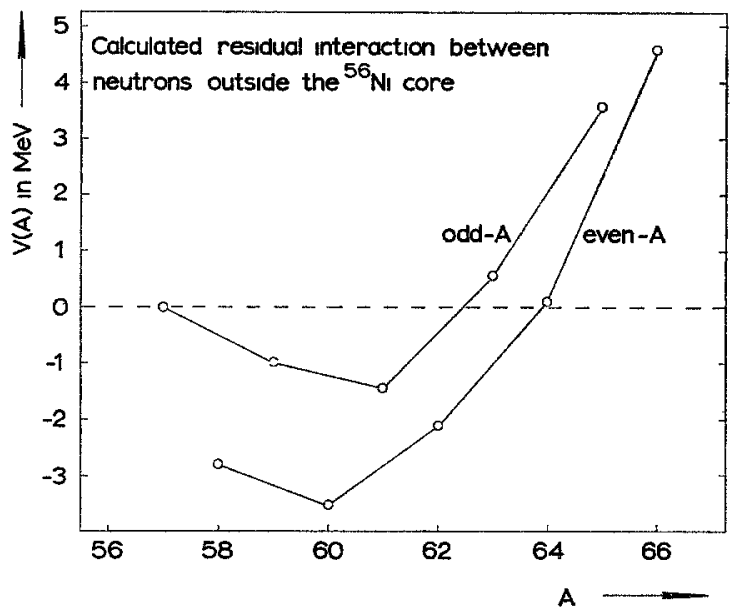

Fig 2. The residual interaction $V(A)$ (see text for a definition) between neutrons outside the ${ }^{56} \mathrm{~N}_{1}$ core for the ground states of $N_{1}$ isotopes as a function of mass number

state binding energies of neighbouring nucles as a function of mass number are shown in fig. 1

The residual interaction between the neutrons outside the ${ }^{56} \mathrm{~N} 1$ core is given by

$$
V(A)=E_{b}(A-56)-\left\langle n\left(\mathrm{p}_{\frac{3}{2}}\right)\right\rangle E\left(\mathrm{p}_{\frac{3}{2}}\right)-\left\langle n\left(\mathrm{f}_{\frac{5}{2}}\right)\right\rangle E\left(\mathrm{f}_{\frac{5}{2}}\right)-\left\langle n\left(\mathrm{p}_{\frac{1}{2}}\right)\right\rangle E\left(\mathrm{p}_{\frac{1}{2}}\right),
$$

where $\langle n(\rho)\rangle$ denotes the average occupation of the orbit $\rho$, as calculated from the present wave functions. The symbols $E_{b}(A-56)$ and $E(\rho)$ denote the binding energy and single-particle energies, respectively, taken with respect to the ${ }^{56} \mathrm{Ni}$ core. The change of the interaction $V(A)$ from attractive (negative) into repulsive around mass 
63 as 1 llustrated in fig. 2 , has also been reported by Lawson et al. ${ }^{3}$ ) and Cohen et al. ${ }^{1}$ ), who used different effective interactions.

Only three parameters are involved in the calculation of excitation energies the $T=1$ strength of the SDI given by $A_{1}$, and the single-particle energy differences

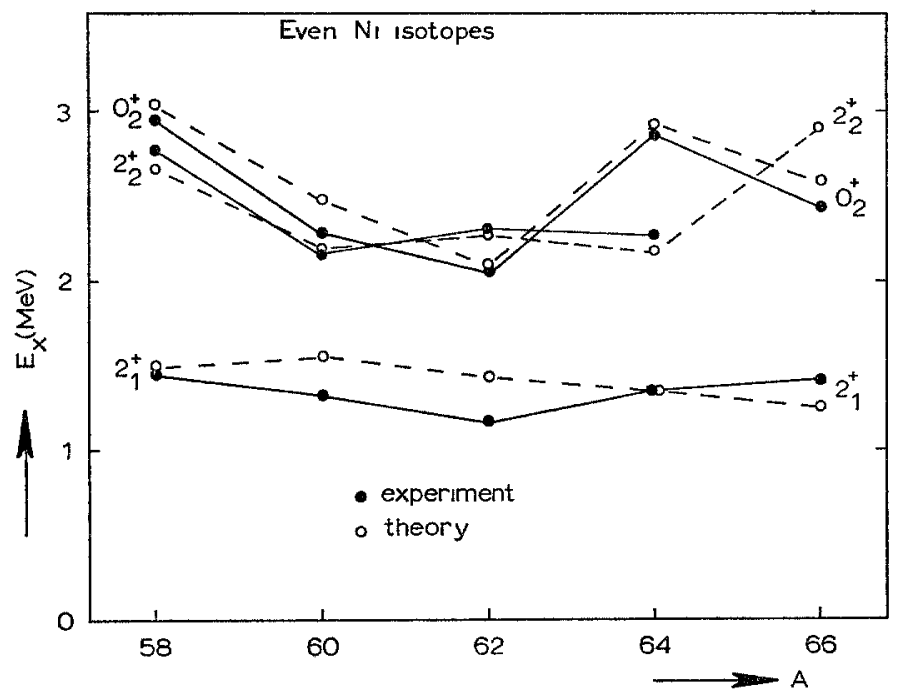

Fig 3. A comparison between theory and experiment for the excitation energies of the first $2^{+}$ and second $0^{+}$and $2^{+}$states in even-mass $\mathrm{Ni}$ isotopes as a function of mass number

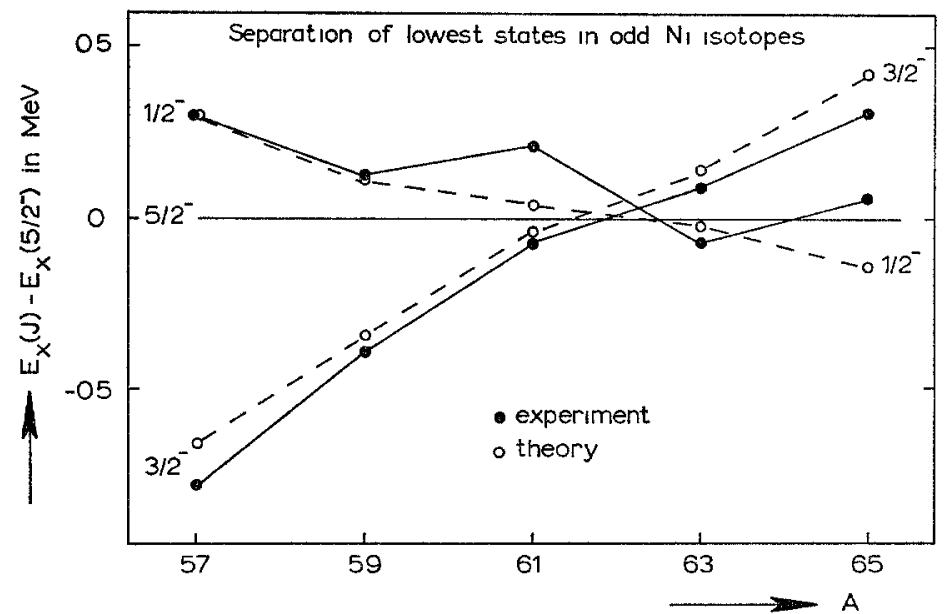

Fig. 4. The separation energies of the lower $\frac{3}{2}^{-}, \frac{5}{2}^{-}$and $\frac{1}{2}^{-}$states in odd-mass $N_{1}$ isotopes as a function of mass number.

$E\left(\mathrm{f}_{\frac{5}{2}}\right)-E\left(\mathrm{p}_{\frac{3}{2}}\right)$ and $E\left(\mathrm{p}_{\frac{1}{2}}\right)-E\left(\mathrm{p}_{\frac{3}{2}}\right)$. The monopole strength $B$ and the absolute singleparticle energies affect only the binding energies. 
The calculated and observed mass dependence of the excitation energies for the lowest two $J^{\pi}=0^{+}$and $J^{\pi}=2^{+}$states are given in fig. 3. It is seen that the relative positions of the second $J^{\pi}=0^{+}$and $2^{+}$states are well reproduced. The separation energies of the lowest $J^{\pi}=\frac{3}{2}^{-}, \frac{5}{2}^{-}$and $\frac{1}{2}^{-}$and states in the odd-mass N1 1sotopes are given in fig. 4 , as a function of mass number.

The calculated and observed energy levels for $A=58-66 \mathrm{~N}_{1}$ isotopes are compared in fig. 5. The average absolute deviation between calculated and observed excitation energies of the 36 levels used in the fit is $0.11 \mathrm{MeV}$. The present excitation energies calculated with the SDI agree much better with experiment than those obtained from shell-model and quasiparticle calculations with renormalized Kuo-Brown reaction matrix elements, as reported by Rustgi et al. ${ }^{8}$ ) and for ${ }^{59} \mathrm{Ni}$ by Singh et al. ${ }^{7}$ ). In fact the agreement with experiment is even better than in refs. ${ }^{1,2}$ ), although in their calculations more parameters are used to obtain values of two-body matrix elements.

\section{Electromagnetic properties}

\subsection{THE LEAST-SQUARES FITTING PROCEDURE}

The theory underlying the use of effective electromagnetic operators and the fitting procedure by which the empirical effectrve values are obtained has already been discussed in detal in ref. ${ }^{17}$ ). The effective charge $e_{\mathrm{n}}$ for the E2 and the effective reduced single-particle matrix elements for both the E2 and M1 operators were obtained from least-squares fits to avallable experimental data. The relative signs of the experimental transition amplitudes, which are needed ${ }^{17}$ ) in the fitting procedure, are assumed to be equal to the signs calculated from the wave functions with bare nucleon $g$-factors and a positive neutron charge.

The experimental errors $\Delta$ have been used in the least-squares fits, except for some cases where larger errors are taken according to the following rules:

(1) $\Delta \geqq 0.005$ and 0.5 W.u. for M1 and $\mathrm{E} 2$ transitions, respectively;

(i1) $\Delta \geqq 25 \%$ for both M1 and E2 transitions;

(iii) $\Delta \geqq 5 \%$ for the magnetic dipole moments.

With these rules the fit is focussed more on the relatively strong transitions and takes into account the generally accepted uncertainty in life-time measurements.

Only the lowest two excited states of a given spin and parity in each nucleus have been taken into account in the fitting procedure.

\subsection{ELECTRIC QUADRUPOLE TRANSITIONS AND MOMENTS}

The effective neutron charge $e_{\mathrm{n}}=(170 \pm 0.08) e$ was determined from a leastsquares fit to the experimental data of $33 \mathrm{E} 2$ transition strengths (11 of which are upper limits) and three quadrupole moments. Separate fits for the nuclei ${ }^{58} \mathrm{~N}_{1},{ }^{60} \mathrm{Ni}$ and ${ }^{62} \mathrm{Ni}$ yielded, within the uncertainty of the calculation, the same value of $e_{\mathrm{n}}$.

An independent adjustment of five reduced single-particle matrix elements for the E2 operator did not reproduce the experimental data much better than the one-param- 

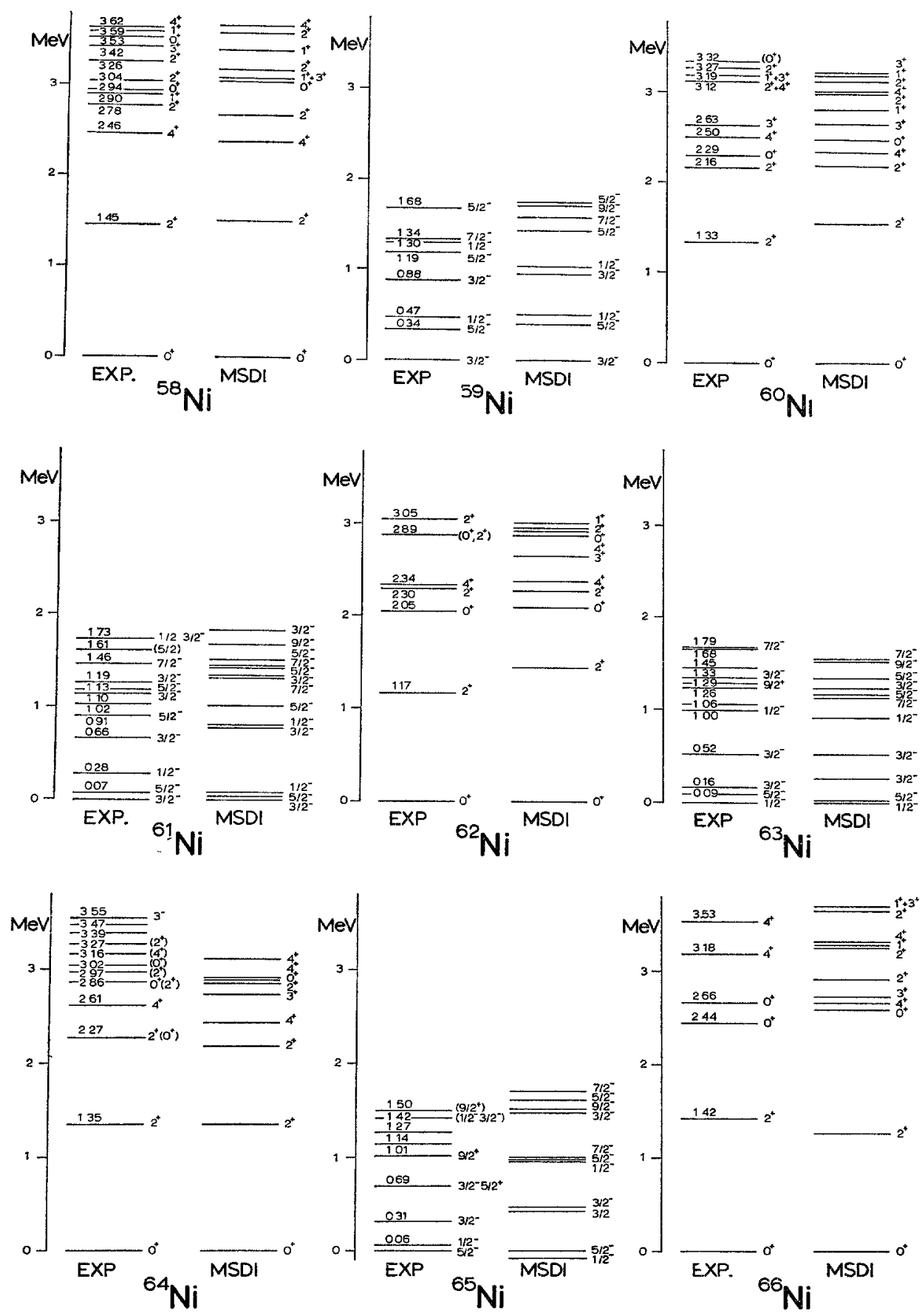

Fig. 5. Comparison between calculated and observed level schemes of $A=58-66$ Ni usotopes For references to expermental data see caption of figs $6-14$. 
eter fit of the neutron charge mentioned above. Therefore the final results have been calculated with one (orbit-independent) effective neutron charge. The average absolute deviation between experiment and theory for the 19 strongest E2 transitions (1-15 W.u.) 1s 3.5. W.u., while the average measured strength is 7.0 W.u.

The discussion of the quadrupole moments which are given in figs. 6-14, is hampered by the lack of sufficient experimental information and the large uncertainties in the avalable experimental data. The only significant discrepancy between experiment and theory is found in the quadrupole moment of the first $J^{\pi}=2^{+}$state in ${ }^{60} \mathrm{Ni}$.

TABLE 2

Values of M1 reduced single-particle matrix elements (n.m)

\begin{tabular}{|c|c|c|c|c|c|}
\hline Transition & $p_{\frac{3}{2}} \rightarrow p_{\frac{3}{2}}$ & $f_{\frac{5}{2}} \rightarrow f_{\frac{5}{2}}$ & $\mathrm{p}_{\frac{1}{2}} \rightarrow \mathrm{p}_{\frac{1}{2}}$ & $p_{\frac{3}{2}} \rightarrow f_{\frac{s}{2}}$ & $\mathrm{p}_{\frac{3}{2}} \rightarrow \mathrm{p}_{\frac{1}{2}}$ \\
\hline (I) bare-nucleon value & -341 & +274 & +1.08 & 0 & -3.05 \\
\hline (II) effective value & -161 & +1.03 & +1.91 & +0.58 & -1.35 \\
\hline ratio II/I $\left\{\begin{array}{l}\text { this work } \\
\text { Rimin }^{a} \text { ) }\end{array}\right.$ & $\begin{array}{l}0.47 \\
0.53\end{array}$ & $\begin{array}{l}0.38 \\
0.41\end{array}$ & $\begin{array}{l}1.77 \\
0.85\end{array}$ & $\begin{array}{l}\infty \\
\infty\end{array}$ & $\begin{array}{l}0.44 \\
0.42\end{array}$ \\
\hline
\end{tabular}

a) Ref. ${ }^{18}$ ).

\subsection{MAGNETIC DIPOLE TRANSITIONS AND MOMENTS}

Five effective reduced single-particle matrix elements are obtained from a leastsquares fit to the experimental data of $30 \mathrm{M} 1$ transition strengths (13 of which are upper limits) and two dipole moments. These effective matrix elements, together with the bare-nucleon values are given in table 2 . The ratios between the presently determined M1 matrix elements and the bare-nucleon values agree, except for one case, very well with those calculated with perturbation theory ${ }^{18}$ ) as shown in table 2 .

A possible mass dependence of the effective M1 matrix elements could not be studied because of the scarcity of experimental data. Good reproduction of the observed transition strengths could not be obtained by adjusting the neutron spin and orbital $g$ factors, $g_{s}$ and $g_{l}$. This is probably due to the vanishing (l-forbidden) $\mathrm{p}_{\frac{3}{2}} \rightarrow \mathrm{f}_{\frac{s}{2}}$ matrix element for any value of the effective $g$-factors. It is seen from table 2 that the empirically determined value of this matrix element differs signuficantly from zero. Moreover the $p_{\frac{3}{2}} \rightarrow f_{\frac{5}{2}}$ single-particle transition contributes strongly to several M1 strengths; e.g. the M1 strengths of the $0.76 \rightarrow 0 \mathrm{MeV}$ transition in ${ }^{57} \mathrm{~N} 1$, the $294 \rightarrow$ $2.90 \mathrm{MeV}$ and $2.90 \rightarrow 0 \mathrm{MeV}$ transitions $1{ }^{58} \mathrm{~N}_{1}$ are completely determined by this single-particle transition Therefore the final results have been calculated with the effective reduced single-particle matrix elements. The average absolute deviation between experiment and theory for the 15 strongest M1 transitions $(0.01-0.14$ W.u. $)$ is $0.03 \mathrm{~W}$.u., while the average measured strength is 0.07 W.u.

The calculated magnetic dipole moments for ${ }^{61} \mathrm{Ni}$ given in fig. 10 agree excellently with experiment. The discrepancy found in the dipole moment of the first excited 


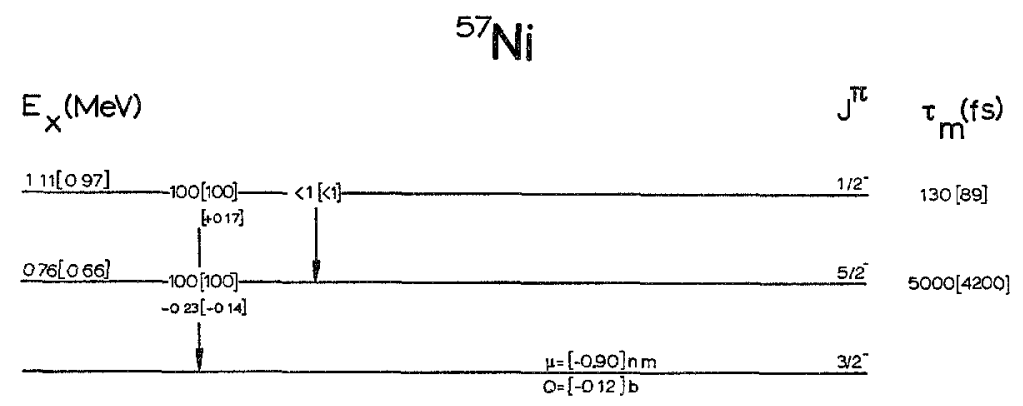

Fig 6. Comparison between calculated [in square brackets] and measured quantities for ${ }^{57} \mathrm{~N}_{1}$ The experimental data have been taken from refs ${ }^{19,20}$ ) For further detals see caption of fig 7

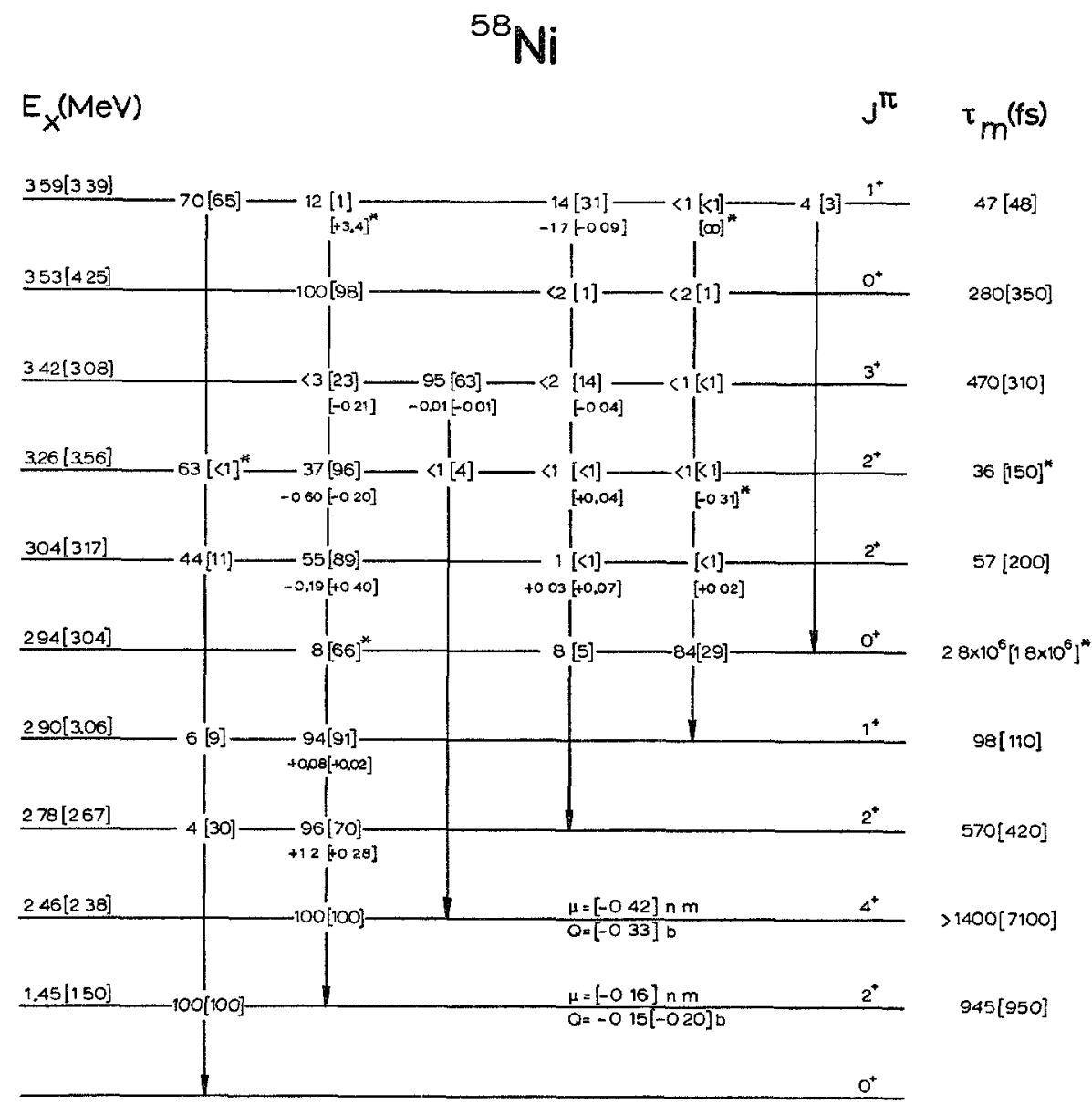

Fig 7. Comparison between calculated [in square brackets] and measured quantities for ${ }^{58} \mathrm{Ni}$. The expermental data have been taken from refs $21-27$ ) An additıonal $(5 \%) 3.42 \rightarrow 3.04 \mathrm{MeV}$ transition bas been reported in refs. ${ }^{21,23}$ ). Calculated quantities with uncertainties larger than a factor of two (see text) are indicated by an asterisk. The excitation energies are given in a distorted scale. The signs of the E2/M1 mixing ratios are all given in the phase convention of Rose and Brink ${ }^{51}$ ). 
$J^{\pi}=\frac{5}{2}^{-}$state in ${ }^{63} \mathrm{~N} 1$ (see fig. 12) cannot be removed by changing the effective M1 matrix elements, since the theoretical expressions for the dipole moments of the first $J^{\pi}=\frac{5}{2}^{-}$states in ${ }^{61} \mathrm{~N} 1$ and ${ }^{63} \mathrm{~N} 1$ turn out to be almost identical.

\section{${ }^{59} \mathrm{Ni}$}
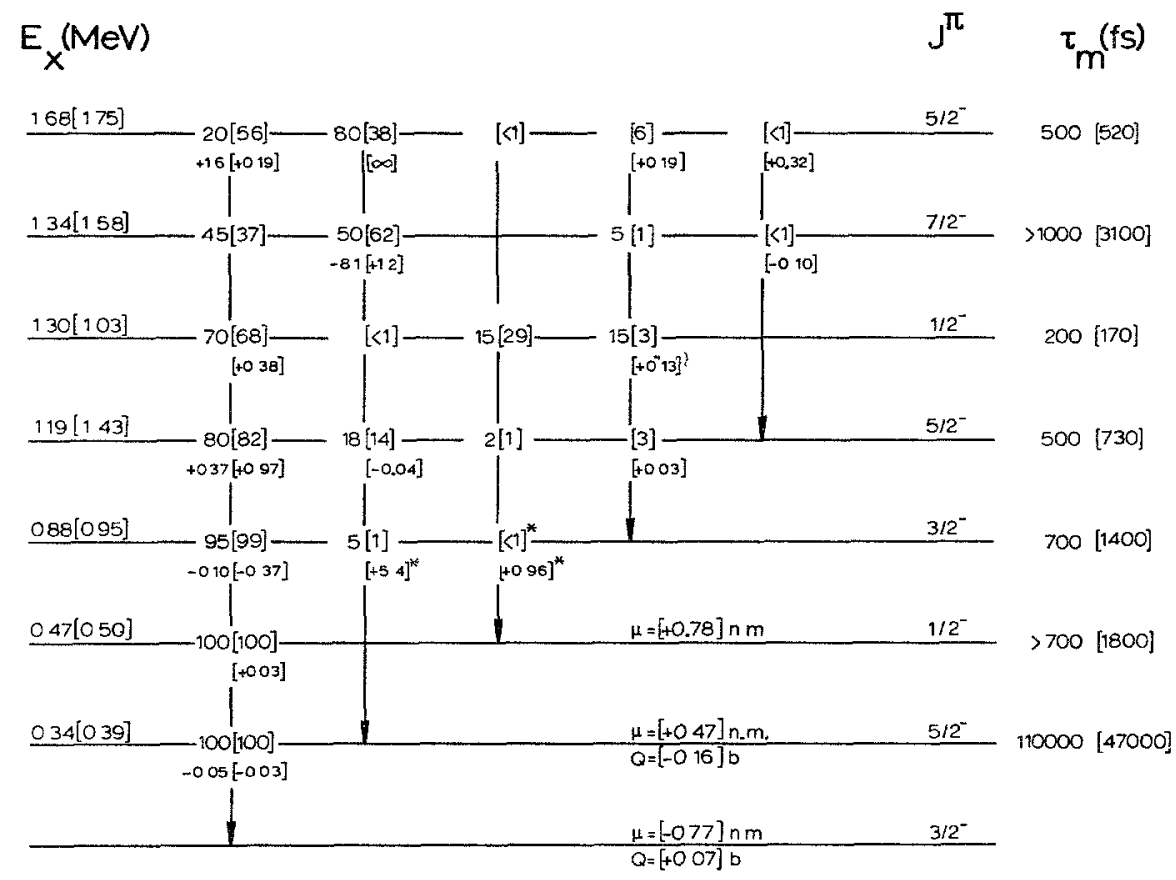

Fig 8. Comparison between calculated [in square brackets] and measured quantitres for ${ }^{59} \mathrm{~N}_{1}$ The experimental data have been taken from refs. ${ }^{30-32}$ ) and from the prelimmary results of refs. ${ }^{28,29}$ ). The electromagnetic transition data have not been used in the least-squares fittung procedure For further details see caption of fig. 7 .

\subsection{LIFETIMES, BRANCHING AND MIXING RATIOS}

The observable quantities for the isotopes ${ }^{57-65} \mathrm{~N}_{1}$ obtained from calculated transition strengths and experimental transition energies are compared with the data in figs $6-14$. The signs of the calculated mixing ratios follow from the relative signs of the E2 and M1 amplitudes. The phase convention of Rose and Brink ${ }^{51}$ ) has been used throughout.

Assuming the model to be correct, the uncertaintres in the calculated quantities are in general expected to be smaller than $25 \%$ In a few cases the errors are possibly larger than a factor of two. There are two effects thay may yield calculated quantities with the larger errors. One of them is the computational inaccuracy of the Racah algebra involved, which affects only M1 strengths calculated to be less than $10^{-4}$ W.u. and E2 strengths less than $10^{-2}$ W.u. Another uncertainty, affectnng only some 
M1 strengths, results from the errors in the experımental input data and thus from the inaccuracy of the parameters. All cases with errors possibly larger than a factor of two are indicated in figs. $6-14$ by asterisks.

\section{${ }^{60} \mathrm{Ni}$}

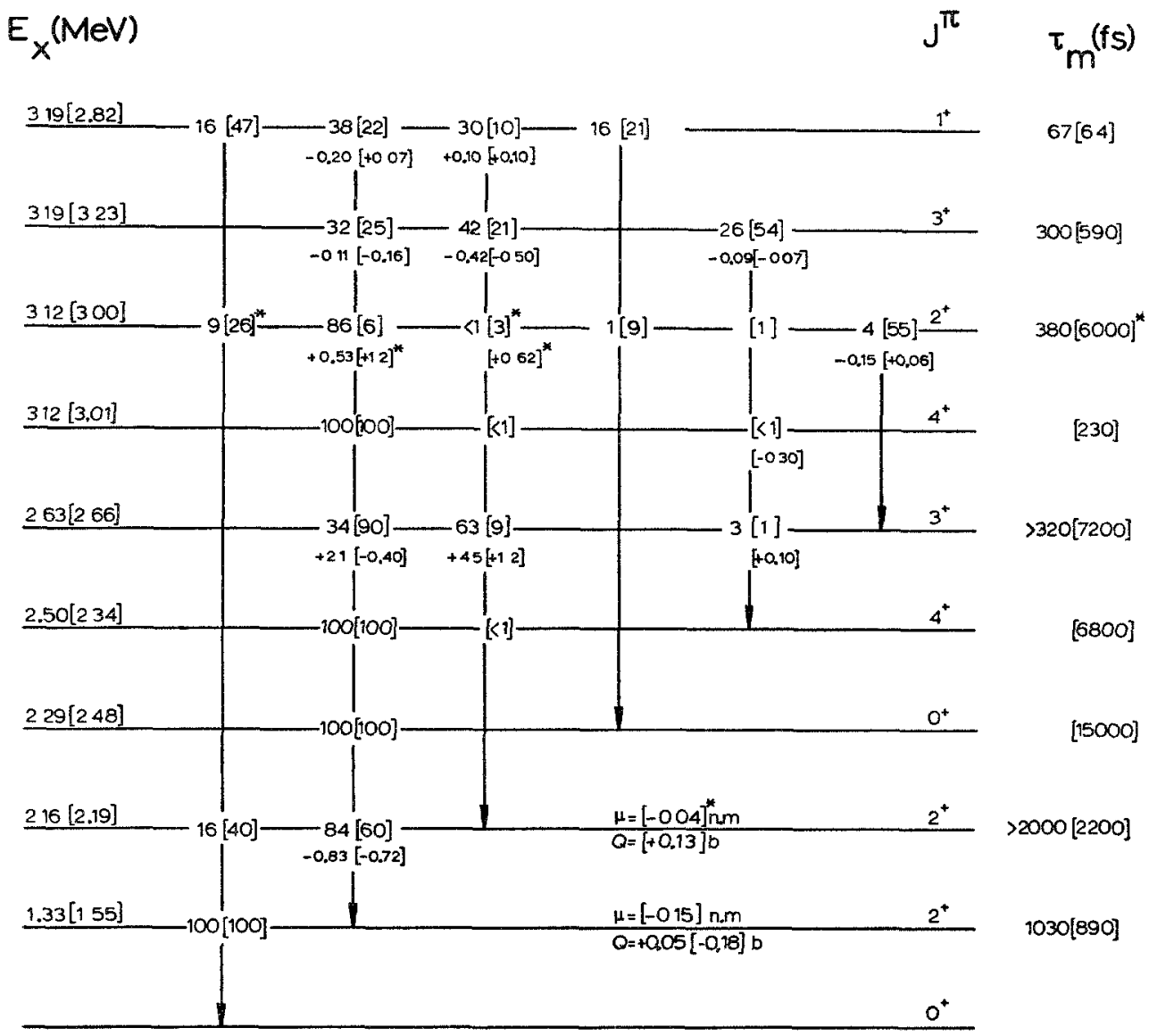

Fig 9 Comparison between calculated [in square brackets] and measured quantities for ${ }^{60} \mathrm{~N}_{1}$. The experimental data have been taken from refs $22,26,27,33-38$ ). For further detarls see caption of fig. 7

A meaningful systematic calculation of the electromagnetic properties of ${ }^{66} \mathrm{Ni}$ is not possible since the level scheme is insufficiently known. The lifetimes of the first $J^{\pi}=2^{+}, 0^{+}$and $4^{+}$excited states are calculated to be $1950,850 \times 10^{3}$ and $330 \mathrm{fs}$, respectively. 


\section{${ }^{61} \mathrm{Ni}$}

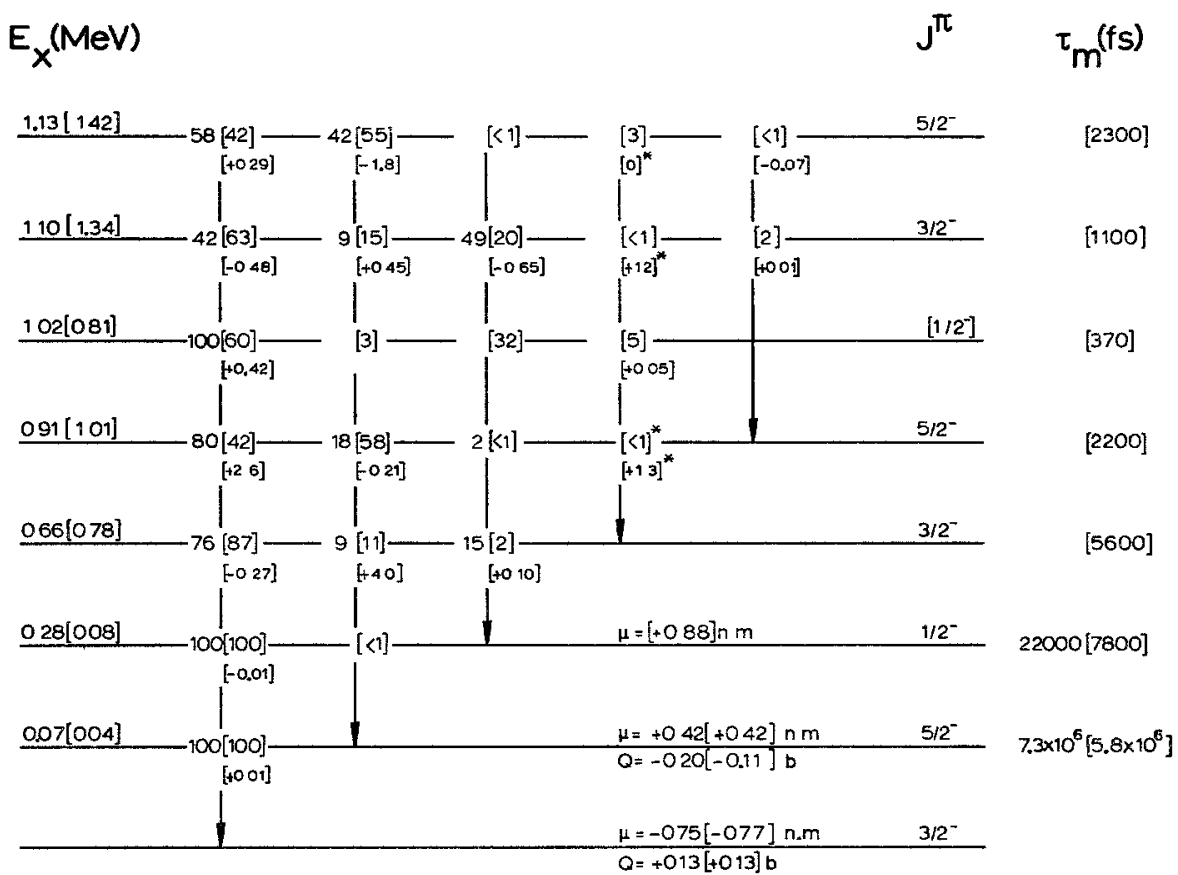

Fig. 10 Comparison between calculated [in square brackets] and measured quantities for ${ }^{61} \mathrm{~N}$. The experimental data have been taken from refs ${ }^{31,39-43}$ ). For further detals see caption of fig 7

\section{${ }^{62} \mathrm{Ni}$}

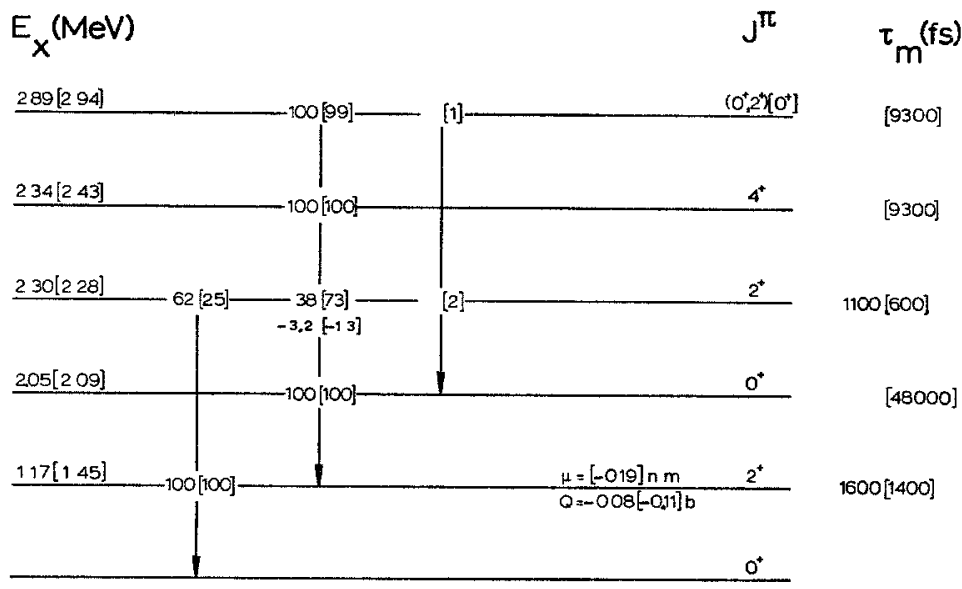

Fig. 11. Comparison between calculated [1n square brackets] and measured quantities for ${ }^{62} \mathrm{Nl}$. The experimental data have been taken from refs. $22,26,36,37,44,45$ ). For further detals see caption of fig 7 . 


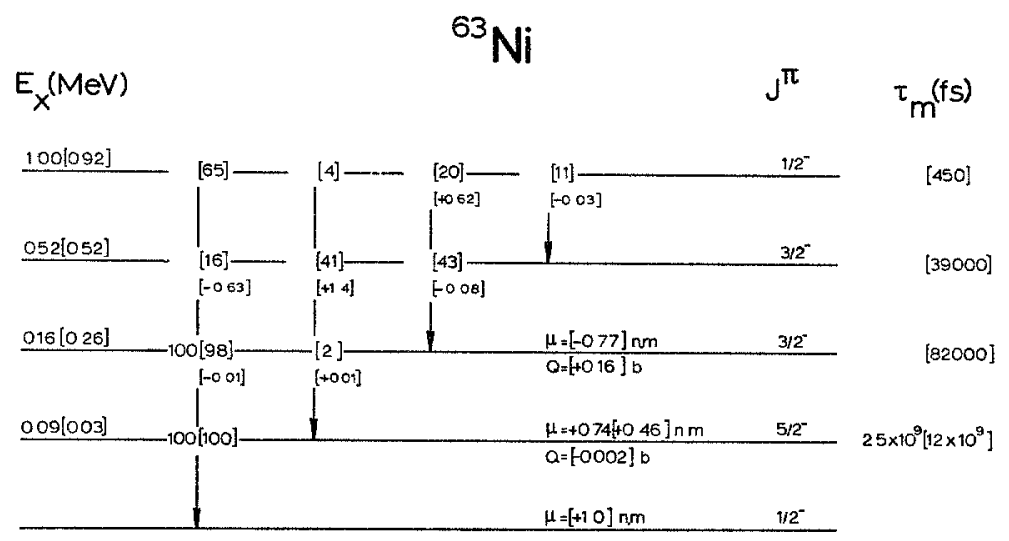

Fig. 12. Comparison between calculated [in square brackets] and measured quantities for ${ }^{63} \mathrm{~N}_{1}$ The experimental data have been taken from refs $41,46-49$ ). For further detals see caption of fig 7

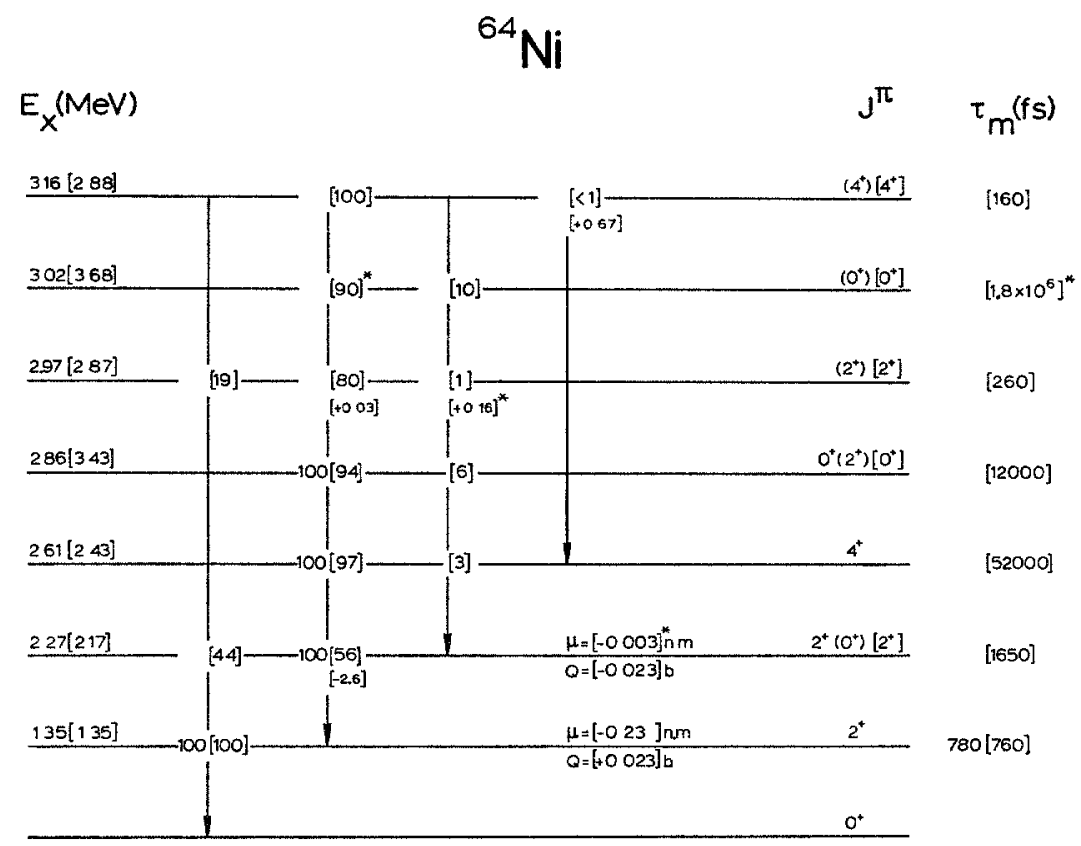

Fig. 13. Comparison between calculated [in square brackets] and measured quantities for ${ }^{64} \mathrm{Ni}_{\mathrm{i}}$ The experimental data have been taken from refs. ${ }^{36,45,46}$ ). For further detalls see caption of fig 7

\section{Discussion}

Three empirıcally determined parameters suffice to reproduce simultaneously the level schemes of ten Ni 1sotopes. Two additional parameters are involved in the calculation of the binding energies. The agreement with experıment is good, especially in view of the simplicity of the interaction, the limited configuration space and the 
neglect of ${ }^{56} \mathrm{~N}$ core excitations. For example the calculated states in ${ }^{58} \mathrm{Ni}$ can be related with known levels up to the tenth excited state, while only two active neutrons are taken into account.

Good results for $\mathrm{E} 2$ and $\mathrm{M} 1$ transition rates in nine $\mathrm{N}_{1}$ 1sotopes are obtaned from least-squares fits to the experimental data in the complete mass region. Only one effective neutron charge, but five effective reduced M1 single-particle matrix elements

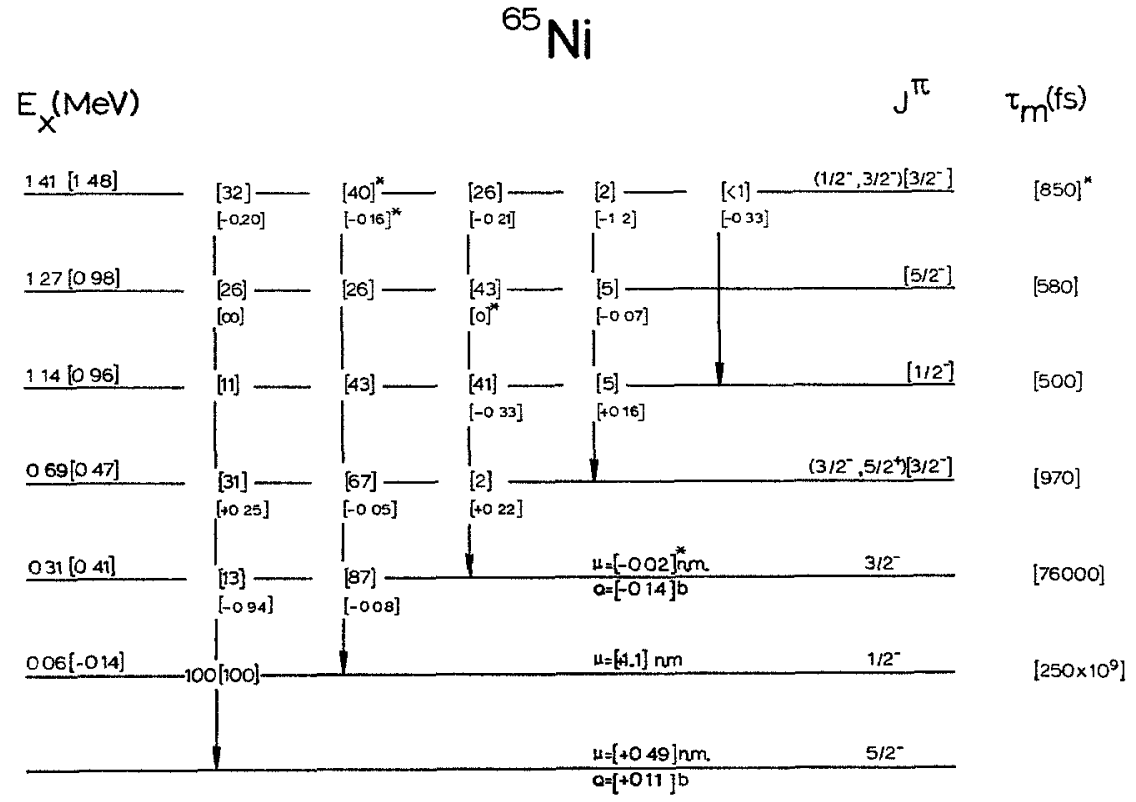

Fig 14 Comparison between calculated [in square brackets] and measured quantities for ${ }^{65} \mathrm{Ni}$. The experimental data have been taken from refs. ${ }^{47,48,50}$ ). For further detals see caption of fig 7

are fitted as parameters. A general indication of the agreement between experiment and theory in connection with electromagnetic properties is provided by a comparison of nuclear lifetimes. The results of the present calculations for ${ }^{58} \mathrm{~N}$ and ${ }^{59} \mathrm{Ni}$ (see figs. 7 and 8) are nice, especially if one considers the fact that the experimental data for ${ }^{59} \mathrm{Ni}$ have not been used in the fits because they became avallable after the calculations were finished. The only significant discrepancies that cannot be explained by the uncertainties discussed in subsect. 3.4 occur in the lifetimes for ${ }^{61} \mathrm{~N}_{1}\left(\frac{1}{2}^{-}\right)_{1}$ and ${ }^{63} \mathrm{~N}_{1}\left(\frac{5}{2}^{-}\right)_{1}$. Unfortunately a lack of experimental information prevents further comparison.

Some general features of calculated transition rates observed also in similar investigations ${ }^{17,52}$ ) in sd shell nucle can be summarized as follows:

(1) Electromagnetic properties for the third or higher state with given spin and parity that occurs in a particular nucleus are generally poorly reproduced, indicating unrehable wave functions for these states. 
(11) Calculated very small transition rates with $\mathrm{M} 1$ strengths less than $10^{-4} \mathrm{~W} \mathrm{u}$. or E2 strengths less than $10^{-2}$ W.u. may contain large errors due to inaccuracies in the wave functions, in the computations or in the experimental imput data and thus in the parameters of the effective operators.

These effects can be nicely observed for ${ }^{58} \mathrm{Ni}$ (see fig. 7), where several observables for the levels at $E_{\mathrm{x}}=2.94,3.04$ and $326 \mathrm{MeV}$ are poorly reproduced.

TABLE 3

The ratio $B\left(\mathrm{E} 2,2_{2}{ }^{+} \rightarrow 0_{1}{ }^{+}\right) / B\left(\mathrm{E} 2 ; 2_{2}{ }^{+} \rightarrow 2_{1}{ }^{+}\right)$for even-mass N1 1sotopes

\begin{tabular}{|c|c|c|c|c|c|}
\hline$\overbrace{\text { Ref. }} A$ & 58 & 60 & 62 & 64 & 66 \\
\hline $\left.\exp ^{37}\right)$ & 0002 & 0003 & 0.04 & & \\
\hline this work & 0.2 & 001 & 002 & 001 & 3 \\
\hline 1) & 30 & 0.05 & 0.03 & & \\
\hline 2) & 60 & 002 & 001 & 0.01 & 1 \\
\hline$\left.{ }^{6}\right)$ & 20 & 01 & 0.0003 & 0.06 & \\
\hline 8) & 05 & 0.7 & & & \\
\hline${ }^{26}$ ) set $A$ & 0.3 & 9 & 0.01 & & \\
\hline set $B$ & 05 & 3 & 6 & & \\
\hline
\end{tabular}

The observed inhibited $2_{2}^{+} \rightarrow 0_{1}^{+}$cross-over transitions are compared with experlment and with the results of other calculations in table 3. It is seen that this inhibition is better reproduced by the present calculation than by other shell-model calculations [refs $1,2,6,8,26)$ ] for which different interactions were employed However, for the $2_{2}^{+} \rightarrow 0_{1}^{+}$cross-over transition in ${ }^{58} \mathrm{~N}$, there is still a large discrepancy between theory and experıment, probably due to the absence of seniority $v=4$ components in the present wave function Such components in ${ }^{58} \mathrm{~N}_{1}$ wave functions would necessarily imply core excitations, which are not allowed in the present model. In line with the strong $1 \mathrm{f}_{\frac{7}{2}}$ admixture in the wave function of the ${ }^{56} \mathrm{Ni}$ ground state ${ }^{14}$ ), $4 \mathrm{p}-2 \mathrm{~h}$ configurations may play a major role $1 \mathrm{n}^{58} \mathrm{Ni}$.

In contrast with calculations of refs. ${ }^{1,2,6}$ ) the present wave function of the $0_{2}^{+}$ state in ${ }^{62} \mathrm{~N}_{1}$ contains predominantly $(90 \%)$ seniority $v=4$ and 6 components, indicating a two-phonon character. This is not found for $\mathrm{O}_{2}^{+}$states in the other evenmass Ni-1sotopes, which are mainly composed of $v=0$ components. The second $2^{+}$ states are found to consist for more than $90 \%$ of components with $v=2$, except again in ${ }^{62} \mathrm{~N}_{1}$ where this state contains $60 \% v=4$ components

It may be concluded that in comparison with the various reported interactions ${ }^{1-10}$ ) the presently applied modıfied surface delta interaction is quite successful. Further improvement of the results may be expected by taking into account particle excitations from the $1 \mathrm{f}_{\frac{7}{2}}$ orbit. In the first place, however, more experimental information is required, especially on mixing ratios and lifetımes for Ni isotopes with $A \geqq 60$, on branching ratios for $\mathrm{N}_{1}$ isotopes with $A \geqq 62$ and on the level scheme of ${ }^{66} \mathrm{~N}_{1}$. 
The authors are much indebted to the Oliver Lodge Laboratory (Liverpool) for their generous offer to use the computer facilities. We would also like to thank J.F.A. van Hienen and R. E. Horstman for their help. This work was performed as part of the Stichtıng voor Fundamenteel Onderzoek der Materie (F.O.M.) with financial support from the Nederlandse Organısatie voor Zuiver-Wetenschappelyk Onderzoek (Z W.O.).

\section{References}

1) S Cohen, R. D Lawson, M. H. Macfarlane, S P. Pandya and M Soga, Phys. Rev. 160 (1967) 903

2) N. Auerbach, Phys Rev 163 (1967) 1203

3) R D Lawson, M. H. Macfarlane and T. T S. Kuo, Phys. Lett 22 (1966) 168

4) R Arvieu, O Bohigas and C Quesne, Nucl. Phys A143 (1970) 577

5) A Plastino, R Arvieu and S A Moszkowski, Phys Rev 145 (1966) 837

6) L S Hsu, Nucl Phys A96 (1967) 624

7) R P Singh, R. Raj, M. L Rustgi and H W Kung, Phys. Rev. C2 (1970) 1715

8) M L Rustgi, H W Kung, R Raj, R. A. Nisley and M. H Hull, Jr, Phys Rev C4 (1971) 854

9) B Roj, R Raj and M. L. Rustgi, Phys. Rev. C1 (1970) 207

10) Y. K Gambhir and R. Ray, Phys. Rev 161 (1967) 1125

11) M. R. Gunye and S B Khadkıkar, Nucl Phys A165 (1971) 508

12) H. Chandra and M L Rustg1, Phys Rev C4 (1971) 406

13) P W. M. Glaudemans, P. J Brussaard and B. H W1ldenthal, Nucl. Phys A102 (1967) 593

14) S. S. M. Wong and W G Davies, Phys Lett. 28B (1968) 77

15) P W M Glaudemans, Proc 4th Summer school on nuclear physics, vol 2 (Rudziska, Poland, 1971) p 183

16) A. H Wapstra and N. B. Gove, Nucl. Data 9 (1971) 267

17) P W M Glaudemans, P M. Endt and A. E L. Dieperink, Ann. of Phys 63 (1971) 134

18) A. Rumm, Theory of nuclear structure, Trieste lectures, 1969, p. 713

19) C R Gould, D P Balamuth, P. F. Hunruchsen and R. W. Zurmuhle, Phys. Rev 188 (1969) 1792

20) C R. Could, E C Hagen, R. V Poore, N. R. Roberson, G E Mitchell and D R Tilley, Phys. Rev Lett 25 (1970) 463

21) D F. H Start, R. Anderson, L E. Carlson, A. G. Robertson and M A Grace, Nucl Phys. A162 (1971) 49

22) M. C. Bertın, N Benczer-Koller, G. G. Seaman and J. R. MacDonald, Phys Rev 183 (1969) 964

23) D. M. van Patter, R N Horoshko, H. L. Scott and P. F Hinrichsen, Nucl Phys A137 (1969) 353

24) R. N. Horoshko, P. F. Hinrichsen, L. W. Swenson and D M van Patter, Nucl. Phys A104 (1967) 113

25) P. F. Hinnchsen, G T Wood and S M Shafroth, Nucl. Phys. 81 (1966) 449

26) P M. S. Lesser, D. Cline and J. D. Purvis, Nucl Phys. A151 (1970) 257

27) P. M. S. Lesser, D. Cline, A Bahnsen, C. K. Cline and R N. Horoshko, Bull. Am. Phys Soc. 16 (1971) 12

28) J. L Eberhardt, Utrecht Univ, private communication

29) N R Robertson, Durham Univ., private communication

30) J. W. Tepel, J. G. Malan and J. A. M. de Villers, Nucl Phys. A158 (1970) 129

31) F. Stecher-Rasmussen, thesis, Utrecht Unıv., 1971

32) J. A. Aymar, H R. Hiddleston, S. E. Darden and A. A. Rollefson, Bull Am. Phys Soc. 16 (1971) 1184

33) C. Moazed, T. Becker, P. A. Assimakopoulos and D M van Patter, Nucl Phys A169 (1971) 651

34) F Rauch, D. M. van Patter and P. F. Hinrichsen, Nucl. Phys A124 (1969) 145

35) P Beuzit, Saclay, private communication

36) W. Darcey, R Chapman and S Hinds, Nucl. Phys. A170 (1971) 253

37) D $M$ van Patter, E. J. Hoffman, T Becker and P. A. Assimakopoulos, Nucl. Phys. A178 (1972) 355 
38) D Cline, H S Gertzman, H E. Gove, P M S Lesser and J. J Schwartz, Nucl. Phys A133 (1969) 445

39) E N Shipley, R E. Holland and F. J. Lynch, Phys. Rev 182 (1969) 1165

40) J. Vervier, Nucl Data B2-5 (1968) 81

41) J Kopecký, K Abrahams and F Stecher-Rasmussen, Nucl Phys. A188 (1972) 535

42) G. H Fuller and V. W. Cohen, Nucl. Data A5 (1969) 433

43) J Gorng, $Z$ Naturf. 26a (1971) 1931

44) D. M van Patter, D Neuffer, H L Scott, C Mozaed and P F Hinnchsen, Nucl Phys. A146 (1970) 427

45) P Beuzit, J Delaunay, J. P Fouan and N Cindro, Nucl Phys A128 (1969) 594

46) H. Verheul, Nucl. Data B2-3 (1968) 31

47) T R Anfinsen, K Bjørndal, A Graue, J R. Lien, G. E Sandvık, L. O. Tveita and K. Ytterstad, Nucl Phys. A157 (1970) 561

48) I. M Turkicwicz, P. Beuzit, J Delaunay and J P Fouan, Nucl Phys A143 (1970) 641

49) J. Bleck, R Michaelsen, W Ribbe and W Zeitz, Phys. Lett 32B (1970) 41

50) P. Roussel, G Bruge, A. Bussiere, H Faraggi and J. E. Testoni, Nucl. Phys. A155 (1970) 306

51) H J Rose and D. M Brink, Revs Mod Phys 39 (1967) 306

52) $M J$ J A. de Volgt, P. W M. Glaudemans, J de Boer and B. H Wildenthal, Nucl. Phys. A186 (1972) 365

53) I. S. Hsu and J B French, Phys. Lett 19 (1965) 135 